\title{
A polymorphism within the connective tissue growth factor (CTGF) gene has no effect on non-invasive markers of beta-cell area and risk of type 2 diabetes
}

\author{
Olga Pivovarova ${ }^{\mathrm{a}, \mathrm{b}, *}$, Eva Fisher $^{\mathrm{c}}$, Katarzyna Dudziak $^{\mathrm{d}}$, Iryna Ilkavets ${ }^{\mathrm{e}}$, Steven Dooley, \\ Petr Slominsky ${ }^{\mathrm{f}}$, Svetlana Limborska ${ }^{\mathrm{f}}$, Martin O. Weickert ${ }^{\mathrm{a}, \mathrm{b}, \mathrm{g}}$, Joachim Spranger ${ }^{\mathrm{a}, \mathrm{b}}$, \\ Andreas Fritsche $^{\mathrm{d}}$, Heiner Boeing ${ }^{\mathrm{c}}$, Andreas F. H. Pfeiffer ${ }^{\mathrm{a}, \mathrm{b}}$ and Natalia Rudovich ${ }^{\mathrm{a}, \mathrm{b}}$ \\ ${ }^{a}$ Department of Clinical Nutrition, German Institute of Human Nutrition, Potsdam-Rehbruecke, Nuthetal, Germany \\ ${ }^{\mathrm{b}}$ Department Endocrinology, Diabetes and Nutrition, Charité University, Medicine, Campus Benjamin Franklin, \\ Berlin, Germany \\ ${ }^{\mathrm{c}}$ Department of Epidemiology, German Institute of Human Nutrition, Potsdam-Rehbruecke, Nuthetal, Germany \\ ${ }^{\mathrm{d}}$ Division of Endocrinology, Diabetology, Angiology, Nephrology, and Clinical Chemistry, Department of Internal \\ Medicine, University Hospital, Tübingen and Eberhard Karls University Tübingen, Member of the German, Center \\ for Diabetes Research (DZD), Tübingen, Germany \\ ${ }^{\mathrm{e}}$ Molecular Hepatology - Alcohol Associated Diseases, Medical Clinic II, Faculty of Medicine Mannheim at \\ Heidelberg University, Mannheim, Germany \\ ${ }^{\mathrm{f}}$ Institute of Molecular Genetics, Russian Academy of Sciences, Moscow, Russia \\ ${ }^{\mathrm{g}}$ Warwickshire Institute for the Study of Diabetes, Endocrinology and Metabolism, University Hospitals Coventry \\ and Warwickshire, Coventry, UK and Clinical Sciences Research Institute, Warwick Medical School, University of \\ Warwick, Coventry, UK
}

\begin{abstract}
Chromosomal locus 6q23 is strongly linked to type 2 diabetes (T2DM) and related features including insulin secretion in various ethnic populations. Connective tissue growth factor (CTGF) gene is an interesting T2DM candidate gene in this chromosome region. CTGF is a key mediator of progressive pancreatic fibrosis up-regulated in type 2 diabetes. In contrast, $C T G F$ inactivation in mice compromises islet cell proliferation during embryogenesis. The aim of our study was to investigate an impact of $C T G F$ genetic variation on pancreatic beta-cell function and T2DM pathogenesis. We studied the effect of a common $C T G F$ polymorphism rs 9493150 on the risk of the T2DM development in three independent German cohorts. Specifically, the association between $C T G F$ polymorphism and non-invasive markers of beta-cell area derived from oral glucose tolerance test was studied in subjects without diabetes. Neither in the Metabolic Syndrome Berlin Potsdam (MESYBEPO) study $(n=1026)$ $(\mathrm{OR}=0.637, \mathrm{CI}(0.387-1.050) ; p=0.077)$ nor in the European Prospective Investigation into Cancer and Nutrition-Potsdam (EPIC-Potsdam) $(n=3049)$ cohort $(\mathrm{RR}=0.77 \mathrm{CI}(0.49-1.20), p=0.249$ for the recessive homozygote in general model), a significant association with increased diabetes risk was observed. The risk allele of rs9493150 had also no effect on markers of beta-cell area in the combined analysis of the MESYBEPO and Tübingen Family Study $(n=1826)$. In conclusion, the polymorphism rs9493150 in the 5'-untranslated region of the CTGF gene has no association with T2DM risk and surrogate markers of beta-cell area.
\end{abstract}

Keywords: Genetic association, connective tissue growth factor gene, C-peptide, blood glucose, beta-cell mass, type 2 diabetes mellitus

${ }^{*}$ Corresponding author: Dr. Olga Pivovarova, German Institute of Human Nutrition Potsdam, Arthur-Scheunert-Allee 114-116, 14558
Nuthetal, Germany. Tel.: +49 332008 8749; Fax: +49 332008 8777; E-mail: olga.pivovarova@dife.de. 


$\begin{array}{ll}\text { Abbreviations } & \\ \text { CTGF } & \text { Connective tissue growth factor } \\ \text { IFG } & \text { impaired fasting glucose } \\ \text { IGT } & \text { impaired glucose tolerance } \\ \text { MESYBEPO } & \text { Metabolic Syndrome Berlin- } \\ & \text { Potsdam study } \\ \text { NGT } & \text { normal glucose tolerance } \\ \text { OGTT } & \text { oral glucose tolerance test } \\ \text { OR } & \text { odds ratio } \\ \text { SNP } & \text { single nucleotide polymorphism } \\ \text { T2DM } & \text { type 2 diabetes mellitus } \\ \text { TÜF } & \text { Tübingen Family Study }\end{array}$

\section{Introduction}

Chromosomal locus 6q23 is strongly linked to type 2 diabetes (T2DM) and related features including insulin secretion in various ethnic populations [1-3]. Particularly, the family study in nonobese diabetic Finns subjects pointed out the primary $\beta$-cell defect linked with this locus coincident with linkage analysis results for fasting and 2-hour insulin in subjects without diabetes [1]. Ectonucleotide pyrophosphatase phosphodiesterase (ENPP1) is a well characterized positional candidate gene for T2DM at chromosome 6q23 identified in a number of whole-genome association studies [4-6].

Another interesting candidate gene coding the connective tissue growth factor (CTGF) is located $100 \mathrm{~kb}$ distant from diabetes-associated ENPP1 polymorphism K121Q. CTGF is a secreted protein integrating several growth factor-signalling pathways including TGF- $\beta$, Wnt and bone morphogenetic protein signaling $[7,8]$. Loss of CTGF function results in defective islet morphogenesis in animal embryos and leads to a dramatic decrease in beta-cell proliferation at late gestation [8]. Diabetes-induced fibrosis is characterized by elevated $C T G F$ expression in multiple organs including kidney, arteries and heart [9-11], and hyperglycemic hyperinsulinemia induces $C T G F$ gene expression in pancreatic stellate cells [12]. Stellate cells in pancreas, as well as in liver, are involved in the tissue repair, but their persistent activation lead to fibrosis [13]. Pancreatic islet cell fibrosis is one of the major factors leading to progressive $\beta$-cell loss in T2DM [14]. Interestingly, CTGF activates pancreatic stellate cells through a specific integrin receptor [15], and thus may participate in the regulation of the $\beta$-cell mass in T2DM. However, nothing is known about the impact of $C T G F$ genetic variations on pancreatic $\beta$-cell function. Therefore, we investigated the occurrence and potential influence of genetic variation in the CTGF gene on diabetes pathogenesis.

We investigated the association of a common $C T G F$ polymorphism on diabetes risk in the prospective casecohort study of the European Prospective Investigation into Cancer and Nutrition (EPIC)-Potsdam cohort and in an independent cross-sectional Metabolic Syndrome Berlin Potsdam (MESYBEPO) study from the same geographical region of Germany. In addition, the association between the CTGF polymorphism and noninvasive markers of beta-cell area derived from oral glucose tolerance test [16] was investigated in both MESYBEPO and in Tübingen Family Study (TÜF Study).

\section{Subjects and methods}

\subsection{Cohorts}

All studies have been approved by the local ethic authorities: the Ethical Committees of the State of Brandenburg and Berlin (EPIC-Potsdam study) and University of Potsdam and Charité Medical University of Berlin, Germany (MESYBEPO study) and the University of Tübingen (TÜF study).

We performed a case-cohort study within the prospective EPIC-Potsdam cohort involving 27,548 Caucasian volunteers, mainly aged $35-65$ years, from the general population [17]. The baseline examination included anthropometric measurements, blood sampling, a self-administered validated food-frequency questionnaire, and a personal interview on lifestyle habits and medical history. Follow-up questionnaires have been administered every 2 to 3 years to obtain information on current medication and newly developed diseases, including T2DM [18]. All potentially incident cases of diabetes identified in the course of followup were verified by questionnaires mailed to the diagnosing physicians asking about the date and type of diagnosis, and information on diagnostic tests, and kind of treatment carried out. Only subjects with a physicians' diagnosis of T2DM (ICD10:E11) and diagnosis date after the baseline examination were considered as confirmed incident cases of type 2 diabetes. The casecohort selected from the EPIC-Potsdam study population comprised 801 incident diabetes cases confirmed within a mean follow-up time of $7.1 \pm 1.8$ years and a subcohort of 2322 randomly selected persons from the EPIC-Potsdam cohort described in detail before [19]. 
74 incident diabetes cases belonged to the subcohort. Thus, the study comprised 801 incident cases and 2248 non-cases.

In the cross-sectional MESYBEPO study, the baseline examination of participants recruited from the general population of the Berlin-Brandenburg area in Germany included anthropometric measurements, blood sampling, a $75 \mathrm{~g}$ oral glucose tolerance test (OGTT) for $120 \mathrm{~min}$ and a personal interview on lifestyle habits and medical history [20]. In MESYBEPO study, we genotyped $C T G F$ polymorphism in 1026 subjects and restricted the number of subjects to 991 participants regarding followed criteria to avoid the T2DM misclassification: body mass index (BMI) below 50 and age about 35 years. This study included subjects with T2DM ( $n=227$ ), impaired glucose tolerance/impaired fasting glucose (IGT/IFG, $n=299$ ) and normal glucose tolerance (NGT, $n=440$ ). Baseline characteristics of the study population e.g. insulin and C-peptide levels during OGTT, OGTT-derived indexes of insulin secretion and insulin sensitivity are presented in details previously [20].

The TÜF study consists of 1826 non-diabetic subjects with increased risk for T2DM (mainly positive family history of diabetes). All participants underwent an OGTT with measurement of glucose, insulin and Cpeptide levels every 30 minutes during 3 hour. This cohort included 1279 subjects with NGT and 547 subjects with IFG, IGT or both [21].

\subsection{SNP genotyping}

CTGF single nucleotide polymorphism (SNPs) rs9493150 was selected for analysis on the basis of the high heterozygosity (0.340) and localization in the 5 , untranslated region [22; NCBI SNP database, available at http://www.ncbi.nlm.nih.gov/SNP]. Although this polymorphism locates $1.7 \mathrm{~kb}$ from the start codon, upstream from identified transcription factor binding sites of CTGF promoter [23], it might influence basal and induced CTGF expression by the trans-regulatory mechanism.

In the MESYBEPO cohort, we genotyped $C T G F$ polymorphism in 1026 subjects. DNA was extracted from blood cells using Magnasep magnetic beads (Agowa, Berlin, Germany). Genotyping of SNPs was performed using TaqMan assay-on-demand (Applied Biosystems, Forster City, CA, USA) by means of an ABI 7900HT on 384 well plates. Details of genotyping will be given by the authors upon request. The genotype success rate for all SNPs was $>97 \%$, and genotyping error was $\leqslant 0.5 \%$. In the TÜF study, the rs9493150 of the CTGF gene was genotyped using the iPLEX_MALDI-TOF mass spectrometry (Sequenom, San Diego, CA). To monitor the sequenom test reproducibility and accuracy, samples of 25 individuals were bisequenced for the selected SNP with an ABI Prism 310 genetic analyser (Applied Biosystems).

\subsection{Statistical analysis}

In the EPIC-Potsdam cohort, associations of SNPs with T2DM risk were calculated as relative risks (RRs) stratified by age using Cox proportional-hazards regression models modified according to the Barlow method [24] to account for the case-cohort design with adjustment for gender and BMI. In the MESYBEPO study, genotype frequencies for the SNP were tested against diabetes prevalence as a dichotomous trait using logistic regression for the calculation of genotypebased odds ratios (OR) adjusted for age, gender and BMI.

Analysis of quantitative traits was performed in the MESYBEPO and TÜF cohorts separately, following with combined analysis of both populations. For this aim, linear regression analysis was performed. Nonnormally distributed variables were log transformed before analysis. The nominal significance level was 0.05 . Effect sizes for the power $\geqslant 95 \%$ detectable in the combined analyse of the MESYBEPO and Tübingen studies $(n=2598)$ were $\geqslant 8 \%$ in the additive model and $\geqslant 14 \%$ in the dominant model. Power calculation was performed in the additive inheritance model by F-tests and in the dominant inheritance model by two-tailed t-tests ( $\mathrm{G}^{*}$ power software available at http://www.psycho.uni-duesseldorf.de/aap/projects/ gpower/). Statistical analyses were performed with SAS release 9.1 (SAS Institute, Cary, NC) and SPSS 14 (SPSS Inc., Chicago, IL). In the TÜF study JMP 8 (SAS, Cary, NC, USA) was used.

\section{Results}

In all studied cohorts, the CTGF rs9493150 polymorphism was in Hardy-Weinberg equilibrium. The displayed risk allele frequency was similar with data of the NCBI SNP database and with data of previously published study [22]: $26.2 \%$ in the MESYBEPO cohort, $25.8 \%$ in the EPIC-Potsdam cohort (Table 1) and $28.3 \%$ in the TÜF study. 
Table 1

The relation between $C T G F$ rs9493150 and risk of type 2 diabetes in the prospective EPIC-Potsdam cohort and the cross-sectional MESYBEPO study

\begin{tabular}{lllr}
\hline EPIC-Potsdam & Genotype & Relative risk $(95 \% \mathrm{CI})$ & $p$-value \\
\hline & $\mathbf{n}_{\text {non }- \text { cases }} / \mathbf{n}_{\text {cases }}$ & & \\
& GG $(1239 / 447)$ & 1 (reference) & \\
& GC $(817 / 297)$ & $0.96(0.77-1.19)$ & 0.699 \\
& CC $(167 / 54)$ & $0.77(0.49-1.20)$ & 0.249 \\
\hline MESYBEPO & Genotype & Odds ratio $(95 \% \mathrm{CI})$ & p-value \\
\hline & $\mathbf{n}_{\text {control }} / \mathbf{n}_{\text {case }}$ & & \\
& GG $(452 / 126)$ & 1 (reference) & \\
& GC $(253 / 78)$ & $0.93(0.70-1.25)$ & 0.638 \\
& CC $(64 / 18)$ & $0.64(0.39-1.05)$ & 0.077 \\
\hline
\end{tabular}

The subcohort of EPIC-Potsdam was representative for the entire cohort and thus included 74 incident cases. Those were considered as "cases" in Cox regression models accounting for the case-cohort design together with the "external" incident cases of the remainder cohort. Regression models were calculated using adjustment for gender and BMI in EPIC-Potsdam cohort and for age, gender and BMI in MESYBEPO study.

Table 2

The effect of $C T G F$ polymorphism rs9493150 on the OGTT-derived markers of beta-cell area in MESYBEPO and Tübingen (TÜF) studies

\begin{tabular}{lccccc}
\hline Cohort & $\begin{array}{c}\text { C-peptide/ glucose } \\
\text { pmol/mmol }\end{array}$ & \multicolumn{3}{c}{ rs 9493150 } & p-value \\
\cline { 3 - 5 } & $0 \mathrm{~min}$ & $141.3 \pm 59.7$ & $135.1 \pm 57.7$ & $120.5 \pm 47.8$ & $\mathbf{0 . 0 0 4}$ \\
\hline MESYBEPO & $30 \mathrm{~min}$ & $242.4 \pm 95.0$ & $232.3 \pm 106.4$ & $210.9 \pm 96.3$ & $\mathbf{0 . 0 2 4}$ \\
$(n=410 / 266 / 63)$ & $0 \mathrm{~min}$ & $129.9 \pm 59.5$ & $128.5 \pm 55.9$ & $128.4 \pm 66.0$ & 0.426 \\
TÜF & $30 \mathrm{~min}$ & $256.8 \pm 109.3$ & $254.4 \pm 99.4$ & $254.1 \pm 117.6$ & 0.573 \\
$(n=906 / 772 / 12)$ & &
\end{tabular}

Non diabetic subjects (NGT + IGT/IFG) from two German studies were analyzed. Data are adjusted for age, gender and BMI.

In the EPIC-Potsdam cohort no association with T2DM risk was observed $(\mathrm{RR}=0.77 \mathrm{CI}(0.49-1.20)$, $p=0.249$ for the recessive vs. dominant homozygote) (Table 1). In the cross-sectional MESYBEPO study, we observed a trend to relationships with T2DM risk in the analysis of the non diabetic (NGT+IFG/IGT) vs. diabetic subjects (OR 0.64, $95 \%$ CI 0.39-1.05, $p=$ 0.077 for recessive vs. dominant homozygote, with adjustment for age, gender and BMI). After additional adjustment for waist circumference, used as a marker of abdominal obesity, no significant association with T2DM risk were observed in both cohorts (data not shown). Because of the design of the TÜF study, which included individuals without diabetes, no analyse of diabetes risk association was made in this population.

We next examined whether $C T G F$ polymorphism rs9493150 is associated with variations in the Cpeptide-to- glucose ratio which was used in our study as non-invasive marker of pancreatic beta-cell area. The recent study of Meier et al. [16] demonstrated that the C-peptide-to-glucose ratio after oral glucose ingestion or even in the fasting state is a best predictor of pancreatic beta-cell area in comparison to other established indexes of insulin secretion. In the MESYBEPO study, non-diabetic individuals with the CC-genotype had a lower C-peptide/glucose ratios than those with $\mathrm{CG}$ or GG-genotype ( $p=0.005$ for fasting values and $p=$ 0.017 for values of $30 \mathrm{~min}$ after oral glucose loading in the crude model). This relation remained unchanged after adjustment for age, gender and BMI ( $p=0.004$ and $p=0.024$, respectively; Table 2 ) and in the analysis of the whole MESYBEPO study including diabetic individuals (data not shown).

However, in the TÜF study, no significant effect of $C T G F$ polymorphism on the C-peptide/glucose ratio was revealed $(p=0.426$ for fasting values and $p=$ 0.573 for 30 min values after adjustment for age, gender and BMI; Table 2).

Moreover, we performed combined analysis of the MESYBEPO and TÜF studies to test the effect of $C T G F$ polymorphism on C-peptide to glucose ratios (0 min, $30 \mathrm{~min}$ and AUC). Based on the multiple metabolic abnormalities observed in diabetic subjects, we carried out the statistical analysis only in the subcohort of non-diabetic subjects $(n=2598)$. In this subcohort, the risk allele of rs9493150 had no effect 
Table 3

Combined analyse of the effect of CTGF polymorphism on the OGTT-derived markers of beta-cell area in MESYBEPO and Tübingen studies

\begin{tabular}{|c|c|c|c|c|c|c|}
\hline \multirow[t]{2}{*}{ Parameter } & & \multicolumn{3}{|c|}{ rs 9493150} & \multicolumn{2}{|c|}{$p$-value } \\
\hline & & GG & $\mathrm{GC}$ & $\mathrm{CC}$ & Padd & Pdom \\
\hline $\mathrm{N}(\%)$ & & $1338(52 \%)$ & $1046(40 \%)$ & $214(8 \%)$ & & \\
\hline BMI & & $30.6 \pm 8.6$ & $29.9 \pm 8.7$ & $29.2 \pm 7.4$ & & \\
\hline \multirow{3}{*}{$\begin{array}{l}\text { C-peptide/ glucose } 0 \text { min } \\
(\mathrm{pmol} / \mathrm{mmol})\end{array}$} & $\mathrm{NGT}+\mathrm{IGT} / \mathrm{IFG}$ & $134.0 \pm 59.9$ & $130.9 \pm 57.5$ & $126.5 \pm 61.0$ & 0.84 & 0.88 \\
\hline & NGT & $127.3 \pm 55.4$ & $126.1 \pm 56.4$ & $127.5 \pm 64.0$ & 0.49 & 0.26 \\
\hline & IGT/IFG & $147.7 \pm 65.9$ & $140.9 \pm 58.6$ & $124.8 \pm 55.6$ & 0.34 & 0.16 \\
\hline \multirow{3}{*}{$\begin{array}{l}\text { C-peptide/ glucose } 30 \mathrm{~min} \\
(\mathrm{pmol} / \mathrm{mmol})\end{array}$} & $\mathrm{NGT}+\mathrm{IGT} / \mathrm{IFG}$ & $252.8 \pm 105.4$ & $249.0 \pm 101.7$ & $242.1 \pm 113.5$ & 0.30 & 0.48 \\
\hline & NGT & $262.4 \pm 106.2$ & $256.7 \pm 103.6$ & $258.7 \pm 116.2$ & 0.67 & 0.49 \\
\hline & IGT/IFG & $233.2 \pm 101.1$ & $232.4 \pm 95.4$ & $210.4 \pm 101.6$ & 0.45 & 0.87 \\
\hline $\operatorname{AUC}_{\mathrm{C}-\text { peptide }(0-120)} /$ & $\mathrm{NGT}+\mathrm{IGT} / \mathrm{IFG}$ & $317.0 \pm 102.7$ & $317.0 \pm 102.5$ & $312.3 \pm 116.9$ & 0.73 & 0.72 \\
\hline \multirow{2}{*}{$\mathrm{AUC}_{\mathrm{glucose}}(0-120)$} & NGT & $325.5 \pm 103.1$ & $325.5 \pm 103.0$ & $321.4 \pm 120.3$ & 0.71 & 0.74 \\
\hline & IGT/IFG & $299.8 \pm 99.9$ & $300.8 \pm 99.2$ & $295.0 \pm 108.8$ & 0.81 & 0.68 \\
\hline Glucose $_{0} \min , \mathrm{mmol} / \mathrm{l}$ & & $5.1 \pm 0.5$ & $5.1 \pm 0.6$ & $5.1 \pm 0.5$ & 0.23 & 0.72 \\
\hline
\end{tabular}

Non diabetic subjects ( $n=2598$; NGT, $n=1740$ and IGT and/or IFG, $n=858$ ) from two German studies were analyzed. Data are adjusted for age, gender, BMI and centre effect.

on metabolic traits measured in the OGTT (Table 3). Interestingly, analysis of subjects only with impaired glucose homeostasis (IGT and/or IFG) revealed the reduced beta-cell secretion parameters in $\mathrm{CC}$ carriers, but these differences were not statistically significant (Table 3), both before and after adjustment for sex, age, BMI and centre effects. Moreover, homozygous carriers of the $\mathrm{C}$-allele had a trend to the lower BMI (Table 3).

\section{Discussion}

In the present study we investigated potential effect of a common genetic variant in the $C T G F$ gene on the T2DM frequency in two independent German populations. Neither in the cross-sectional MESYBEPO study nor in the prospective EPIC-Potsdam cohort a significant association with increased diabetes risk was found. In addition, the association of OGTT-derived markers of beta-cell area with single genetic marker in $C T G F$ was examined in two German populations from different geographical region. In spite of the observed reduced C-peptide to glucose ratio in the recessive homozygote carriers in the MESYBEPO study, we were unable to identify statistically significant associations of these disturbances with the here studied gene polymorphism in a combined analysis of the MESYBEPO and TÜF populations.

Interestingly, homozygote carriers of C-allele of CTGF SNP demonstrated a trend to lower BMI that might contribute to the finding that no association of the polymorphism with T2DM risk was observed. A comparable effect was described for a GIPR polymorphism by Saxena et al. [25] which was associated with 2-h glucose levels and decreased insulin secretion, but not with T2DM, possibly due to significantly lower BMI in risk allele carriers.

Some limitations of our study need to be mentioned. Firstly, in this study, we focused exclusively on the rs9493150 variant of the CTGF gene. Although we aimed to investigate a clear a priori defined hypothesis, we cannot exclude that other polymorphisms in the genomic region of CTGF might be relevant for T2DM susceptibility and beta-cell area.

Furthermore, in spite of sufficient power to detect moderate changes of beta-cell function, our study might be underpowered to identify smaller alterations. Indeed, the detection of small changes of quantitative diabetic traits (plasma insulin, glucose etc) requires the analysis of very large cohorts including meta-analyses of genome-wide association studies [26]. Therefore, further investigations in considerably larger cohorts are needed to test the influence of genetic variations within locus $6 \mathrm{q} 23$ on pancreatic beta-cell function.

In summary, no association of a common polymorphism in the $C T G F$ gene with T2DM risk was identified in two independent German cohorts. The risk allele of rs9493150 showed no effect on surrogate OGTTderived markers of beta-cell area in the combined analyse of the MESYBEPO and Tübingen populations.

\section{Acknowledgements}

This study was supported by a grant from German Federal Ministry of Education and Research (Grant No. 0313042C/ NR and AFHP) and German Academ- 
ic Exchange Service (DAAD) (OP). The recruitment phase of the EPIC-Potsdam Study was supported by the Federal Ministry of Science, Germany (01 EA 9401) and the European Union (SOC 95201408 05F02). The follow-up of the EPIC-Potsdam Study was supported by the German Cancer Aid (70-2488-Ha I) and the European Community (SOC 98200769 05F02).

We thank all study participants for their cooperation and A. Wagner, S. Grosch, M. Hannemann and K. Sprengel for excellent technical assistance. We gratefully acknowledge Mrs. June Indertal for the English spell check of the manuscript.

\section{Conflict of interest}

The authors declare no conflict of interest.

\section{References}

[1] C. Shtir, I.S. Nagakawa, W.L. Duren et al., Subsets of Finns with high HDL to total cholesterol ratio show evidence for linkage to type 2 diabetes on chromosome 6q, Hum Hered $\mathbf{6 3}$ (2007), 17-25.

[2] R. Duggirala, J. Blangero, L. Almasy et al., A major locus for fasting insulin concentrations and insulin resistance on chromosome $6 \mathrm{q}$ with strong pleiotropic effects on obesityrelated phenotypes in non-diabetic Mexican Americans, Am J Hum Genet 68 (2001), 1149-1164.

[3] K. Xiang, Y. Wang, T. Zheng et al., Genome-wide search for type 2 diabetes/impaired glucose homeostasis susceptibility genes in the Chinese, Diabetes 53 (2004), 228-234.

[4] R. Baratta, P. Rossetti, S. Prudente et al., Role of the ENPP1 K121Q polymorphism in glucose homeostasis, Diabetes 57 (2008), 3360-3364.

[5] K.J. Gaulton, C.J. Willer, Y. Li et al., Comprehensive association study of type 2 diabetes and related quantitative traits with 222 candidate genes, Diabetes 57 (2008), 3136-3144.

[6] J.B. McAteer, S. Prudente, S. Bacci et al., ENPP1 Consortium, The ENPP1 K121Q polymorphism is associated with type 2 diabetes in European populations: evidence from an updated meta-analysis in 42,042 subjects, Diabetes 57 (2008), 11251130 .

[7] S. Mercurio, B. Latinkic, N. Itasaki et al., Connective-tissue growth factor modulates WNT signalling and interacts with the WNT receptor complex, Development 131 (2004), 21372147.

[8] L.A. Crawford, M.A. Guney, Y.A. Oh et al., Connective tissue growth factor (CTGF) inactivation leads to defects in islet cell lineage allocation and beta-cell proliferation during embryogenesis, Mol Endocrinol 23 (2009), 324-336.

[9] S.M. Twigg, Z. Cao, S.V. MCLennan et al., Renal connective tissue growth factor induction in experimental diabetes is prevented by aminoguanidine, Endocrinology 143 (2002), 4907-4915.
[10] R. Candido, K.A. Jandeleit-Dahm, Z. Cao et al., Prevention of accelerated atherosclerosis by angiotensin-converting enzyme inhibition in diabetic apolipoprotein E-deficient mice, Circulation 106 (2002), 246-253.

[11] K.J. Way, K. Isshiki, K. Suzuma et al., Expression of connective tissue growth factor is increased in injured myocardium associated with protein kinase $\mathrm{C}$ beta 2 activation and diabetes, Diabetes 51 (2002), 2709-2718.

[12] O.K. Hong, S.H. Lee, M. Rhee et al., Hyperglycemia and hyperinsulinemia have additive effects on activation and proliferation of pancreatic stellate cells: possible explanation of isletspecific fibrosis in type 2 diabetes mellitus, $J$ Cell Biochem 101 (2007), 665-675.

[13] M.B. Omary, A. Lugea, A.W. Lowe et al., The pancreatic stellate cell: a star on the rise in pancreatic diseases, J Clin Invest 117 (2007), 50-59.

[14] J.W. Kim, S.H. Ko, J.H. Cho et al., Loss of beta-cells with fibrotic islet destruction in type 2 diabetes mellitus, Front Biosci 13 (2008), 6022-6033.

[15] R. Gao and D.R. Brigstock, Connective tissue growth factor $(\mathrm{CCN} 2)$ in rat pancreatic stellate cell function: integrin alpha5beta1 as a novel CCN2 receptor, Gastroenterology 129 (2005), 1019-1030.

[16] J.J. Meier, B.A. Menge, T.G. Breuer et al., Functional assessment of pancreatic beta-cell area in humans, Diabetes $\mathbf{5 8}$ (2009), 1595-1603.

[17] H. Boeing, A. Korfmann and M.M. Bergmann, Recruitment procedures of EPIC-Germany. European Investigation into Cancer and Nutrition, Ann Nutr Metab 43 (1999), 205-215.

[18] M.M. Bergmann, U. Bussas and H. Boeing, Follow-up procedures in EPIC-Germany-data quality aspects. European Prospective Investigation into Cancer and Nutrition, Ann Nutr Metab 43 (1999), 225-234.

[19] M.B. Schulze, H. Al-Hasani, H. Boeing et al., Variation in the HHEX-IDE gene region predisposes to type 2 diabetes in the prospective, population-based EPIC-Potsdam cohort, Diabetologia 50 (2007), 2405-2407.

[20] N. Rudovich, O. Pivovarova, E. Fisher et al., Polymorphisms within insulin degrading enzyme (IDE) gene determine insulin metabolism and risk of type 2 diabetes, J Mol Med 87 (2009), 1145-1151.

[21] M.B. Schulze, K. Hoffmann, H. Boeing et al., An accurate risk score based on anthropometric, dietary, and lifestyle factors to predict the development of type 2 diabetes, Diabetes Care 30 (2007), 510-515.

[22] E. Kovalenko, F. Tacke, O.A. Gressner et al., Validation of connective tissue growth factor (CTGF/CCN2) and its gene polymorphisms as noninvasive biomarkers for the assessment of liver fibrosis, J Viral Hepat 16 (2009), 612-620.

[23] I.E. Blom, A.J. van Dijk, R.A. de Weger et al., Identification of human cen2 (connective tissue growth factor) promoter polymorphisms, Mol Pathol 54 (2001), 192-196.

[24] W.E. Barlow, L. Ichikawa, D. Rosner et al., Analysis of casecohort designs, J Clin Epidemiol 52 (1999), 1165-1172.

[25] R. Saxena, M.F. Hivert, C. Langenberg et al., Genetic variation in GIPR influences the glucose and insulin responses to an oral glucose challenge, Nat Genet 42 (2010), 142-148.

[26] J. Dupuis, C. Langenberg, I. Prokopenko et al., New genetic loci implicated in fasting glucose homeostasis and their impact on type 2 diabetes risk, Nat Genet 42 (2010), 105-116. 


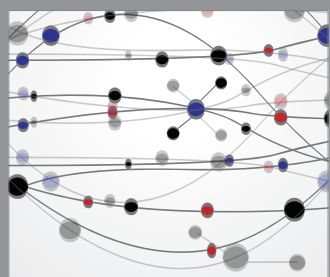

The Scientific World Journal
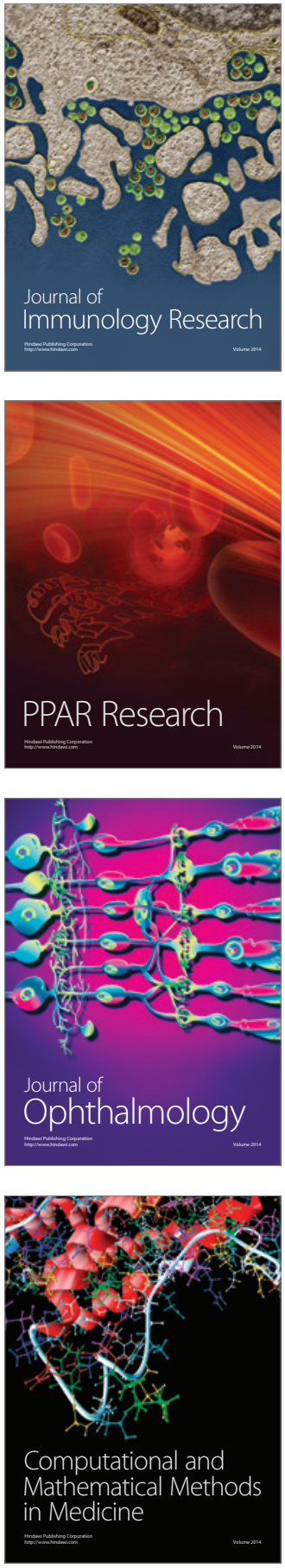

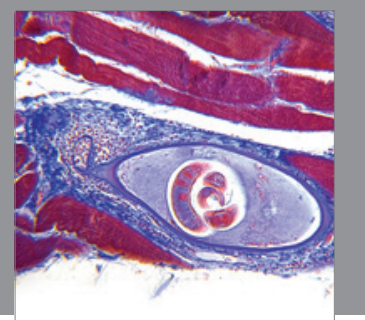

Gastroenterology

Research and Practice
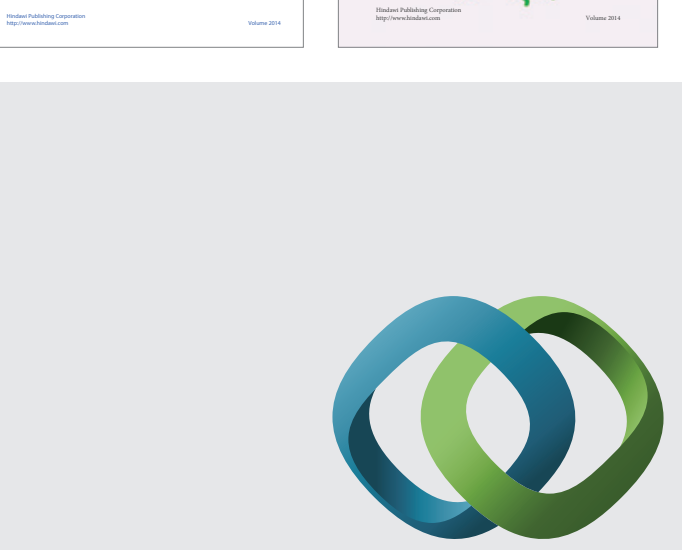

\section{Hindawi}

Submit your manuscripts at

http://www.hindawi.com
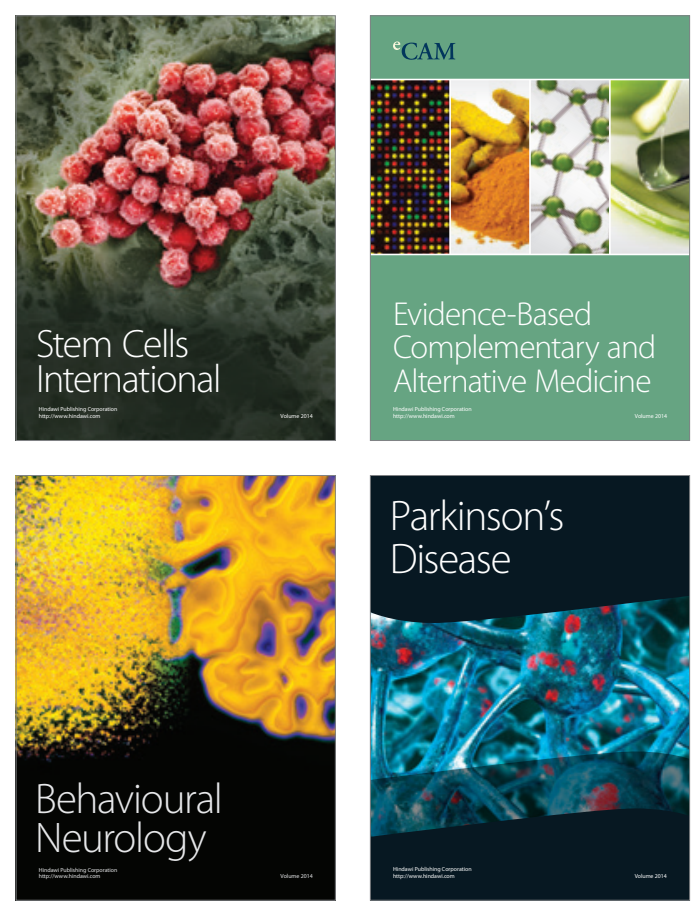

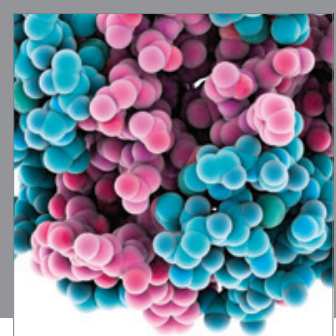

Journal of
Diabetes Research

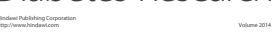

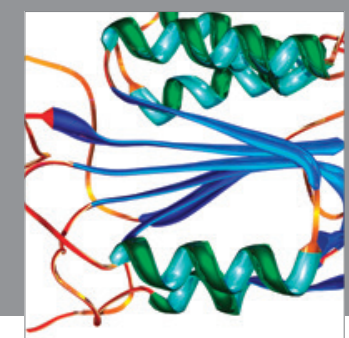

Disease Markers
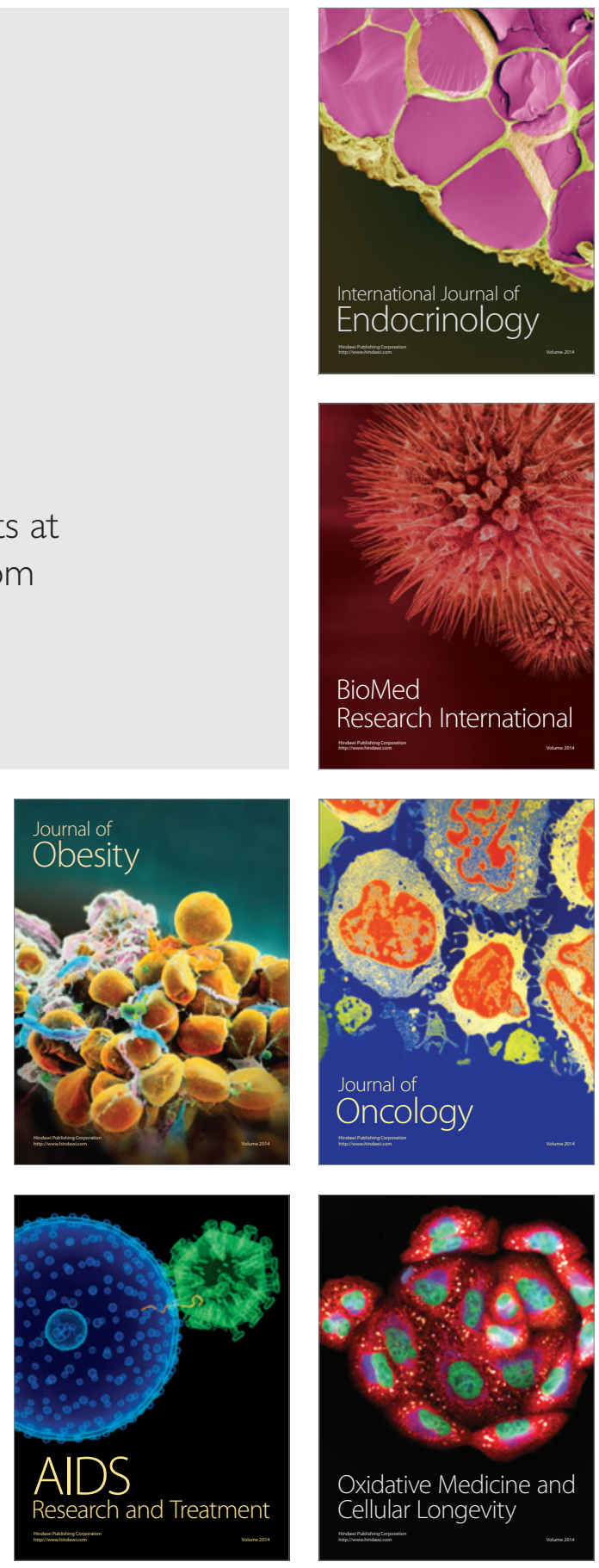